\title{
$\&$ Research Square \\ Predictors of Long-term Right Ventricular Dysfunction in Acute Respiratory Distress Syndrome Survivors
}

\section{Siddharth Dugar ( $\nabla$ dugars@ccf.org )}

Cleveland Clinic Foundation: Cleveland Clinic https://orcid.org/0000-0002-3243-1417

\section{Ryota Sato}

Cleveland Clinic Foundation: Cleveland Clinic

Hari Om Joshi

Parkview Health System Inc: Parkview Medical Center

\section{Xiaozhen Han}

Cleveland Clinic Foundation: Cleveland Clinic

\section{Xiaofeng Wang}

Cleveland Clinic Foundation: Cleveland Clinic

\section{Vasileios Zochios}

University Hospitals of Leicester NHS Trust

\section{Michael Lanspa}

Intermountain Health Care Inc: Intermountain Healthcare

\section{Abhijit Duggal}

Cleveland Clinic Foundation: Cleveland Clinic

\section{Research Article}

Keywords: right ventricular dysfunction, acute cor pulmonale, acute respiratory distress syndrome, acute lung injury, long-term outcomes

Posted Date: September 8th, 2021

DOl: https://doi.org/10.21203/rs.3.rs-864646/v1

License: (c) (i) This work is licensed under a Creative Commons Attribution 4.0 International License.

Read Full License 


\section{Abstract}

Background: Right ventricular (RV) dysfunction develops frequently in patients with acute respiratory distress syndrome and is associated with short-term mortality. However, the incidence of long-term RV dysfunction and risk factor associated with it in ARDS survivors remains unknown. The aim of the study was to assess the prevalence and risk factors of long-term RV dysfunction in ARDS survivors. Method: This is a single-center retrospective cohort study including adult patients with ARDS admitted to the medical intensive care unit (MICU) at Cleveland Clinic between 1/1/2010-12/31/2017. We included patients with ARDS, who had echocardiography performed within 72 hours of MICU admission, survived 90 days from onset of ARDS with post-discharge echocardiography performed between 30-365 days after hospital discharge. RV dysfunction was defined based on the American Society of Echocardiography guidelines. Results: Of the 689 patients who were admitted to Cleveland Clinic for ARDS during the study period, 67 (14.07\%) patients met the study criteria. A total of 34 patients $(50.7 \%)$ had RV dysfunction on post-discharge echocardiogram. In the multivariate logistic regression analyses, the incidence of longterm RV dysfunction was associated only with RVEDA/LVEDA [odds ratio (OR): 589, 95\% confidence interval $(\mathrm{Cl})$ : 71.17-48,400, $\mathrm{p}=0.005$ ]. Conclusion: More than half of ARDS survivors had developed longterm RV dysfunction at post-discharge echocardiogram. Increased ratio of right-to-left ventricular enddiastolic area (RVEDA/LVEDA) ratio during ARDS was the significantly associated with development of long-term RV dysfunction in ARDS survivors. Trial registration: This study was approved by the Institutional Review Committee of Cleveland Clinic (IRB\#19-1200).

\section{Background}

Acute respiratory distress syndrome (ARDS), remains a major cause of admission to the intensive care unit and is associated with significant morbidity and mortality[1] [2]. Advances in management of ARDS have improved survival to hospital discharge [3-5]. Unfortunately, almost a quarter of these ARDS survivors still die within one year of their hospital discharge [6, 7]. These data highlight the need to further investigate and improve not only short-term but also long-term outcomes.

Cardiac dysfunction, and in particular, right ventricular (RV) dysfunction, is increasingly recognized as a predictor of poor clinical outcome in patients with ARDS [8-10]. In a recent meta-analysis, $21 \%$ of patients with ARDS had RV dysfunction, which was associated with increased short-term mortality [11]. Right ventricular dysfunction during critical illness has been shown to be associated with higher longterm mortality [12]. Despite the short-term characterization of RV dysfunction in ARDS, its long-term sequela remains unknown. In the current study we evaluate the incidence and risk factors for long-term RV dysfunction in ARDS survivors.

\section{Methods}

We conducted a single-center retrospective cohort study from January 1, 2010, to May 31, 2017, at a quaternary academic medical center in the United States. We included all adult (>18 years old) patients 
admitted to a medical intensive care unit (MICU) with a diagnosis of ARDS based on Berlin definition, who survived 90 days from MICU admission, and underwent transthoracic echocardiogram (TTE) within 72 hours of admission to MICU with a post-discharge TTE performed between 30 and 365 days after discharge from MICU. Patients were excluded if the only post-discharge TTE available was performed in MICU setting, during evident cardiorespiratory failure, and on invasive mechanical ventilation. We also excluded patients with pre-existing prosthetic cardiac valve, those with moderate to severe chronic obstructive pulmonary disease (COPD), interstitial pulmonary fibrosis (IPF), and acute coronary syndrome within 14 days of either TTE. All patients were identified from the ARDS registry maintained at Cleveland Clinic $[13,14]$. Any additional clinical data were obtained from a review of electronic medical records.

All TTEs were performed by experienced sonographers using commercially available ultrasound systems. All echocardiographic assessments and measurements by board-certified cardiologists were obtained from Syngo Dynamics (Siemens Healthcare, MA, USA) per American Society of Echocardiography and the European Association of Cardiovascular Imaging (ASE/EACVI) recommendations $[15,16]$. In case of missing TTE data, they were collected offline by a diplomate of the National Board of Echocardiography (S.D.). Right Ventricular dysfunction in post-discharge period was defined as per ASE/EACVI guidelines as the presence of any of the following criteria: 1 . Tricuspid annular plane systolic excursion (TAPSE) $<17$ $\mathrm{mm}, 2$. Tricuspid Annulus Peak Velocity $\left(\mathrm{S}^{\prime}\right)<9.5 \mathrm{~cm} / \mathrm{sec}, 3$. Qualitative reduction in RV systolic function and/or Qualitative RV enlargement as identified by board-certified cardiologists, 4. Fractional area contraction (FAC) of RV $<35 \%$ [15-17].

The primary outcome was the one year incidence rate of RV dysfunction in ARDS survivors after discharge. Secondary outcome included: exploration of risk factors for long-term RV dysfunction and associated 1 year mortality from hospital discharge in ARDS survivors. The manuscript was drafted in accordance with The Strengthening the Reporting of Observational Studies in Epidemiology (STROBE) guidelines for reporting observational studies.

\section{Statistical Analysis}

Continuous data were presented as median with interquartile range [IQR], and categorical data were presented as counts with percentage. We compared patients' demographics and echocardiographic parameters using the Mann-Whitney $\mathrm{U}$ test for continuous variables and the Chi-squared test or Fisher's exact test for categorical variables. Missing data imputation for variables was conducted using missForest[18], a robust iterative imputation method based on a random forest, under the assumption of missing-at-random. The reliability of the imputation was checked for the imputed data. Both univariable and multivariable logistic regression analysis were then performed to estimate odds ratios (ORs) and 95\% confidence intervals (Cls) for the incidence of long-term RV dysfunction. For multivariable analysis, we $a$ priori set age, left ventricular ejection fraction (LVEF) during ARDS, TAPSE during ARDS, and right ventricular end-diastolic area/left ventricular diastolic area (RVEDA/LVEDA) during ARDS as the covariates, in order to control for confounding. All analyses were performed using R software (version 4.0; R Foundation for Statistical Computing, Vienna, Austria). A two-tailed p-value of $<0.05$ was considered as 
a statistical significance. This study was approved by the Institutional Review Committee of Cleveland Clinic (IRB\#19-1200).

\section{Results}

Of 689 patients admitted with ARDS to MICU at Cleveland Clinic from 2010-2017, 329 patients were alive at 90 days from admission to MICU. Of these, 67 patients met the inclusion criteria (Fig. 1). The study population included 36 males (53.7\%), with the median age being 57 years. Detailed demographics and treatments of patients with and without long-term RV dysfunction are shown in Table 1. Thirty-four patients $(50.7 \%)$ had RV dysfunction on post-discharge echocardiogram. Of the 36 patients who developed RV dysfunction during ARDS, 23 (63.9\%) had long-term RV dysfunction in the post-discharge echocardiogram, compared to 11 (35.5\%) of 31 in the group who did not have RV dysfunction during ARDS. We observed no significant differences in demographics, comorbidities, disease presentation or severity, treatments or outcomes between those with and without long-term RV dysfunction. There was no difference in 1-year mortality between patients with and without long-term RV dysfunction $(17.6 \% \mathrm{vs}$. $9.1 \%, p=0.48)$. 
Table 1

Baseline demographics, severity of illness and treatment characteristics

\begin{tabular}{|c|c|c|c|c|}
\hline Demographics of the patients & $\begin{array}{l}\text { All } \\
N=67\end{array}$ & $\begin{array}{l}\text { Long-term RVD } \\
\begin{array}{l}(+) \\
N=34\end{array}\end{array}$ & $\begin{array}{l}\text { Long-term } \\
\text { RVD }(-) \\
\mathbf{N}=33\end{array}$ & $\begin{array}{l}\mathrm{p}- \\
\text { value }\end{array}$ \\
\hline Age & $57(44-67)$ & $63(47-73)$ & $55(41-64)$ & 0.084 \\
\hline Male & $36 / 67$ & $18 / 34$ & $18 / 33$ & 1.000 \\
\hline $\mathrm{BMI}\left(\mathrm{kg} / \mathrm{m}^{2}\right)$ & $\begin{array}{l}32.1(26.4- \\
39.0)\end{array}$ & $\begin{array}{l}31.3(26.5- \\
35.3)\end{array}$ & $\begin{array}{l}33.7(26.3- \\
41.9)\end{array}$ & 0.518 \\
\hline SOFA score & $11(9-15)$ & $11(8-14)$ & $11(9-15)$ & 0.508 \\
\hline Causes of ARDS & $\begin{array}{l}48 / 67 \\
(71.6 \%)\end{array}$ & $23 / 34(67.6 \%)$ & 25/33 (75.8\%) & 0.590 \\
\hline $\begin{array}{l}\text { Pulmonary } \\
\text { Extra-pulmonary }\end{array}$ & $\begin{array}{l}19 / 67 \\
(28.4 \%)\end{array}$ & $11 / 34(32.4 \%)$ & $8 / 33(24.2 \%)$ & \\
\hline $\begin{array}{l}\text { History of chronic lung disease } \\
\text { (COPD/ILD) }\end{array}$ & $\begin{array}{l}14 / 67 \\
(20.9 \%)\end{array}$ & $9 / 34(26.5 \%)$ & $5 / 33(15.2 \%)$ & 0.369 \\
\hline History of congestive heart failure & $\begin{array}{l}11 / 67 \\
(16.4 \%)\end{array}$ & $5 / 34(14.7 \%)$ & $6 / 33(18.2 \%)$ & 0.752 \\
\hline $\mathrm{P} / \mathrm{F}$ ratio* & $124(85-171)$ & $130(102-171)$ & $119(82-164)$ & 0.348 \\
\hline Oxygenation Index* & $\begin{array}{l}17.0(8.1- \\
24.7)\end{array}$ & $16.7(7.2-22.2)$ & $\begin{array}{l}17.5(8.1- \\
25.3)\end{array}$ & 0.639 \\
\hline Tidal volume $(\mathrm{ml} / \mathrm{kg})^{*}$ & $7.8(6.6-8.4)$ & $7.8(6.8-8.4)$ & $7.8(6.6-8.3)$ & 0.773 \\
\hline $\operatorname{PEEP}\left(\mathrm{cmH}_{2} \mathrm{O}\right)^{*}$ & $12(8-15)$ & $10(8-15)$ & $12(10-15)$ & 0.151 \\
\hline Plateau pressure $\left(\mathrm{H}_{2} \mathrm{O}\right)^{\star}$ & $28(24-30)$ & $28(27-30)$ & $27(23-32)$ & 0.335 \\
\hline $\mathrm{PaCO}_{2}(\mathrm{mmHg})^{*}$ & $44(39-51)$ & $44(37-49)$ & $44(39-53)$ & 0.366 \\
\hline Use of NMB & $\begin{array}{l}21 / 67 \\
(31.3 \%)\end{array}$ & $8 / 34(23.5 \%)$ & 13/33 (39.4\%) & 0.194 \\
\hline $\begin{array}{l}\text { Use of inhaled pulmonary } \\
\text { vasodilators }\end{array}$ & $7 / 67(10.4 \%)$ & $6 / 34(17.6 \%)$ & $1 / 33(3.0 \%)$ & 0.105 \\
\hline Use of PPV & $3 / 67(4.5 \%)$ & $1 / 34(2.9 \%)$ & $2 / 33(6.1 \%)$ & 0.614 \\
\hline Use of recruitment maneuver & $4 / 67(6.0 \%)$ & $3 / 34(8.8 \%)$ & $1 / 33(3.0 \%)$ & 0.614 \\
\hline Use of Vasopressor & $\begin{array}{l}43 / 67 \\
(64.7 \%)\end{array}$ & $22 / 34(64.7 \%)$ & $21 / 33(63.6 \%)$ & 1.000 \\
\hline $\begin{array}{l}\text { Max Norepinephrine dose } \\
(\mathrm{mcg} / \mathrm{kg} / \mathrm{min})\end{array}$ & $\begin{array}{l}0.09(0.0- \\
0.32)\end{array}$ & $0.12(0.0-0.41)$ & $0.06(0.0-0.26)$ & 0.56 \\
\hline
\end{tabular}




\begin{tabular}{|c|c|c|c|c|}
\hline Demographics of the patients & $N=67$ & $\begin{array}{l}\text { Long-term RVD } \\
(+) \\
\mathrm{N}=\mathbf{3 4}\end{array}$ & $\begin{array}{l}\text { Long-term } \\
\text { RVD }(-) \\
\mathbf{N}=33\end{array}$ & $\begin{array}{l}\text { p- } \\
\text { value }\end{array}$ \\
\hline ICU length of stay & $\begin{array}{l}13.0(10.0- \\
20.0)\end{array}$ & $13.5(9.3-20.3)$ & $\begin{array}{l}13.0(10.0- \\
20.0)\end{array}$ & 0.633 \\
\hline Hospital length of stay & $\begin{array}{l}25.0(16.5- \\
31.5)\end{array}$ & $\begin{array}{l}24.5(14.0- \\
32.3)\end{array}$ & $\begin{array}{l}25.0(19.0- \\
30.0)\end{array}$ & 0.581 \\
\hline Ventilator-free days & $\begin{array}{l}16.0(9.5- \\
20.0)\end{array}$ & $\begin{array}{l}17.0(10.0- \\
20.8)\end{array}$ & $\begin{array}{l}16.0(10.0- \\
20.0)\end{array}$ & 0.606 \\
\hline 1-year mortality & $9 / 67(13.4 \%)$ & $6 / 34(17.6 \%)$ & $3 / 33(9.1 \%)$ & 0.48 \\
\hline \multicolumn{5}{|l|}{ BMI: body mass index } \\
\hline \multicolumn{5}{|c|}{ SOFA: sequential organ failure assessment } \\
\hline \multicolumn{5}{|c|}{ COPD: chronic obstructive pulmonary disease } \\
\hline \multicolumn{5}{|l|}{ ILD: interstitial lung disease } \\
\hline \multicolumn{5}{|c|}{ ARDS: acute respiratory distress syndrome } \\
\hline \multicolumn{5}{|c|}{ PEEP: positive end-expiratory pressure } \\
\hline \multicolumn{5}{|l|}{ NMB: neuromuscular blocker } \\
\hline \multicolumn{5}{|l|}{ PPV: prone position ventilation } \\
\hline ICU: intensive care unit & & & & \\
\hline
\end{tabular}

Baseline and repeat echocardiographic parameters of patients with and without long-term RV dysfunction were shown in Table 2. There was no difference in timing of echocardiogram performed between the two groups during ARDS ( $1(1-2)$ vs. $1(0-1)$ days, $p=0.15$ and in post-discharge period (174 $(92-282)$ vs. $210(78-307)$ days, $p=0.78)$ Table 2 . Patients categorized with long-term RV dysfunction had greater RVEDA/LVEDA than those without long-term RV dysfunction ( 0.73 vs $0.55, p=0.02$ ), while other echocardiographic parameters were not statistically different between groups. 
Table 2

a. Echocardiographic parameters in patients during ARDS.

\begin{tabular}{|c|c|c|c|c|}
\hline \multirow[t]{2}{*}{ Echocardiographic parameters } & \multirow{2}{*}{$\begin{array}{l}\text { All } \\
N=67\end{array}$} & \multirow{2}{*}{$\begin{array}{l}\text { Long-term } \\
\text { RVD }(+) \\
\mathrm{N}=\mathbf{3 4}\end{array}$} & \multirow{2}{*}{$\begin{array}{l}\text { Long-term } \\
\text { RVD }(-) \\
\mathbf{N}=33\end{array}$} & \multirow[t]{2}{*}{$\begin{array}{l}\mathrm{p}- \\
\text { value }\end{array}$} \\
\hline & & & & \\
\hline \multicolumn{5}{|c|}{ Baseline echocardiographic parameters during ARDS } \\
\hline LVEF & $\begin{array}{l}58(54- \\
63)\end{array}$ & $55(51-60)$ & $60(55-65)$ & 0.087 \\
\hline Average $\mathrm{E} / \mathrm{e}^{\prime}$ & $\begin{array}{l}11.3(8.9- \\
16.1)\end{array}$ & $\begin{array}{l}11.4(10.1- \\
16.1)\end{array}$ & $\begin{array}{l}11.3(7.3- \\
15.2)\end{array}$ & 0.562 \\
\hline $\mathrm{E} / \mathrm{A}$ & $\begin{array}{l}0.99 \\
(0.83- \\
1.33)\end{array}$ & $\begin{array}{l}0.99(0.85- \\
1.35)\end{array}$ & $\begin{array}{l}1.00(0.82- \\
1.32)\end{array}$ & 0.524 \\
\hline TAPSE (mm) & $\begin{array}{l}17.1(14.0- \\
21.1)\end{array}$ & $\begin{array}{l}15.5(12.0- \\
19.9)\end{array}$ & $\begin{array}{l}18.8(16.4- \\
21.2)\end{array}$ & 0.098 \\
\hline $\mathrm{S}^{\prime}(\mathrm{cm} / \mathrm{sec})$ & $\begin{array}{l}13.0 \\
(11.0- \\
15.0)\end{array}$ & $\begin{array}{l}13.0(9.9- \\
14.9)\end{array}$ & $\begin{array}{l}13.0(12.0- \\
15.2)\end{array}$ & 0.412 \\
\hline Semi-qualitative RV dilatation & $23 / 67$ & $15 / 34$ & $8 / 33$ & 0.123 \\
\hline Semi-qualitative RV systolic impairment & $23 / 67$ & $15 / 34$ & $8 / 33$ & 0.123 \\
\hline FAC (\%) & $\begin{array}{l}40.0 \\
(26.7- \\
50.0)\end{array}$ & $\begin{array}{l}36.0(25.0- \\
50.0)\end{array}$ & $\begin{array}{l}43.5(33.7- \\
50.0)\end{array}$ & 0.331 \\
\hline RVSP (mmHg) & $\begin{array}{l}44.0 \\
(34.0- \\
52.0)\end{array}$ & $\begin{array}{l}48.0(35.5- \\
60.0)\end{array}$ & $\begin{array}{l}38.5(32.3- \\
48.8)\end{array}$ & 0.07 \\
\hline TR Jet Velocity (m/sec) & $\begin{array}{l}3.0(2.6- \\
3.4)\end{array}$ & $\begin{array}{l}3.0(2.7- \\
3.6)\end{array}$ & $\begin{array}{l}2.8(2.5- \\
3.3)\end{array}$ & 0.16 \\
\hline RVEDA & $\begin{array}{l}19.2 \\
(16.1- \\
23.5)\end{array}$ & $\begin{array}{l}20.2(17.5- \\
24.5)\end{array}$ & $\begin{array}{l}19.0(15.5- \\
22.7)\end{array}$ & 0.395 \\
\hline LVEDA & $\begin{array}{l}29.8 \\
(24.4- \\
35.2)\end{array}$ & $\begin{array}{l}27.9(23.2- \\
33.5)\end{array}$ & $\begin{array}{l}31.5(26.2- \\
35.6)\end{array}$ & 0.080 \\
\hline RVEDA/LVEDA & $\begin{array}{l}0.60 \\
(0.50- \\
0.80)\end{array}$ & $\begin{array}{l}0.73(0.54- \\
0.1 .00)\end{array}$ & $\begin{array}{l}0.55(0.50- \\
0.63)\end{array}$ & 0.020 \\
\hline $\begin{array}{l}\text { Time from onset of ARDS to echocardiogram } \\
\text { (days) }\end{array}$ & $1(0-2)$ & $1(1-2)$ & $1(0-1)$ & 0.158 \\
\hline
\end{tabular}




\begin{tabular}{|c|c|c|c|c|}
\hline Echocardiographic parameters & $\begin{array}{l}\text { All } \\
N=67\end{array}$ & $\begin{array}{l}\text { Long-term } \\
\text { RVD }(+) \\
\mathrm{N}=\mathbf{3 4}\end{array}$ & $\begin{array}{l}\text { Long-term } \\
\text { RVD }(-) \\
\mathbf{N}=\mathbf{3 3}\end{array}$ & $\begin{array}{l}\mathrm{p}- \\
\text { value }\end{array}$ \\
\hline LVEF & $\begin{array}{l}58(51- \\
62)\end{array}$ & $55(50-61)$ & $59(55-65)$ & 0.046 \\
\hline Average $\mathrm{E} / \mathrm{e}^{\prime}$ & $\begin{array}{l}11.2(9.0- \\
14.4)\end{array}$ & $\begin{array}{l}12.5(9.6- \\
14.9)\end{array}$ & $\begin{array}{l}9.5(7.9- \\
12.4)\end{array}$ & 0.562 \\
\hline$E / A$ & $\begin{array}{l}0.98 \\
(0.76- \\
1.30)\end{array}$ & $\begin{array}{l}0.95(0.78- \\
1.31)\end{array}$ & $\begin{array}{l}1.00(0.76- \\
1.23)\end{array}$ & 0.848 \\
\hline TAPSE (mm) & $\begin{array}{l}19.0 \\
(14.0- \\
23.0)\end{array}$ & $\begin{array}{l}14.7(12.3- \\
20.5)\end{array}$ & $\begin{array}{l}20.9(19.0- \\
25.9)\end{array}$ & $<.001$ \\
\hline $\mathrm{S}^{\prime}(\mathrm{cm} / \mathrm{sec})$ & $\begin{array}{l}11.9(9.0- \\
13.9)\end{array}$ & $\begin{array}{l}9.0(8.0- \\
11.9)\end{array}$ & $\begin{array}{l}13.0(12.1- \\
15.3)\end{array}$ & $\begin{array}{l}< \\
0.001\end{array}$ \\
\hline Semi-qualitative RV dilatation & $14 / 67$ & $14 / 34$ & $0 / 33$ & $\begin{array}{l}< \\
0.001\end{array}$ \\
\hline Semi-qualitative RV systolic impairment & $14 / 67$ & $14 / 34$ & $0 / 33$ & $<.001$ \\
\hline FAC (\%) & $\begin{array}{l}40.0(37.0- \\
47.3)\end{array}$ & $\begin{array}{l}40.0(30.7- \\
44.0)\end{array}$ & $\begin{array}{l}42.0(38.5- \\
49.0)\end{array}$ & 0.049 \\
\hline RVSP (mmHg) & $\begin{array}{l}37.0(31.0- \\
48.8)\end{array}$ & $\begin{array}{l}38.5(33.5- \\
54.0)\end{array}$ & $\begin{array}{l}35.0(23.5- \\
37.8)\end{array}$ & 0.039 \\
\hline RVEDA & $\begin{array}{l}17.8 \\
(14.8- \\
23.8)\end{array}$ & $\begin{array}{l}19.7(15.3- \\
29.7)\end{array}$ & $\begin{array}{l}16.9(14.4- \\
21.8)\end{array}$ & 0.131 \\
\hline LVEDA & $\begin{array}{l}30.6 \\
(27.4- \\
36.0)\end{array}$ & $\begin{array}{l}30.0(27.0- \\
35.5)\end{array}$ & $\begin{array}{l}30.7(28.5- \\
37.5)\end{array}$ & 0.325 \\
\hline RVEDA/LVEDA & $\begin{array}{l}0.57(0.5- \\
0.70)\end{array}$ & $\begin{array}{l}0.64(0.50- \\
0.85)\end{array}$ & $\begin{array}{l}0.54(0.50- \\
0.59)\end{array}$ & 0.023 \\
\hline $\begin{array}{l}\text { Time from onset of ARDS to post-discharge } \\
\text { echocardiogram (days) }\end{array}$ & $\begin{array}{l}182(82- \\
291)\end{array}$ & $\begin{array}{l}174(92- \\
282)\end{array}$ & $\begin{array}{l}210(78- \\
307)\end{array}$ & 0.787 \\
\hline \multicolumn{5}{|l|}{$\mathrm{RV}$ : right ventricle } \\
\hline \multicolumn{5}{|l|}{ ARDS: acute respiratory distress syndrome } \\
\hline \multicolumn{5}{|c|}{ TAPSE: tricuspid annular plane systolic excursion } \\
\hline \multicolumn{5}{|l|}{$\mathrm{S}^{\prime}$ : pulsed tissue Doppler S wave } \\
\hline FAC: fractional area contraction & & & & \\
\hline
\end{tabular}




\begin{tabular}{|c|c|c|c|c|}
\hline \multirow[t]{2}{*}{ Echocardiographic parameters } & All & $\begin{array}{l}\text { Long-term } \\
\text { RVD (+) }\end{array}$ & $\begin{array}{l}\text { Long-term } \\
\operatorname{RVD}(-)\end{array}$ & $\begin{array}{l}\mathrm{p}- \\
\text { value }\end{array}$ \\
\hline & $N=67$ & $N=34$ & $N=33$ & \\
\hline \multicolumn{5}{|c|}{ RVSP: right ventricular systolic pressure } \\
\hline \multicolumn{5}{|c|}{ RVEDA/LVEDA: right ventricular end-diastolic area/Left ventricle end diastolic area } \\
\hline
\end{tabular}

In the multivariate logistic regression analyses, the incidence of long-term RV dysfunction was associated only with RVEDA/LVEDA ratio [odds ratio (OR): 589, 95\% confidence interval (CI): 71.17-48,400, $p=0.005$ ], while other covariates did not show significant association, as shown in Table 3. In univariate logistic regression analysis, various definitions of RV dysfunction used in clinical practice were found to be statistically significant and associated with increasing odds of developing long term RV dysfunction, as shown in Table 4. 1) RV dysfunction per ASE guidelines [odds ratio (OR): $3.22,95 \%$ confidence interval (CI): 1.18-8.76, $p=0.022$ ] 2) RVEDA/LVEDA > 0.6 + Septal dyskinesia [odds ratio (OR): 4.18, 95\% confidence interval $(\mathrm{Cl}): 1.43-12.21, p=0.009$ ] and 3) RVEDA/LVEDA $\geq 1$ [odds ratio (OR): $5.58,95 \%$ confidence interval $(\mathrm{Cl}): 1.10-28.20, p=0.038]$. 
Table 3

Logistic regression of predictors for long-term right ventricular dysfunction

\begin{tabular}{|lll|}
\hline Variables & \multicolumn{2}{l}{ Multivariate } \\
\cline { 2 - 3 } Predictors & OR (95\%Cl) & p-value \\
\hline Age & $1.01(0.97-1.05)$ & 0.533 \\
\hline BMI & $0.99(0.94-1.05)$ & 0.845 \\
\hline LVEF (\%) & $0.99(0.94-1.03)$ & 0.560 \\
\hline Average E/e' & $1.06(0.96-1.17)$ & 0.228 \\
\hline P/F ratio on the day of echo & $1.00(0.99-1.01)$ & 0.608 \\
\hline PEEP on the day of echo & $0.96(0.82-1.13)$ & 0.616 \\
\hline RVFAC/RVSP & $1.39(0.29-6.75)$ & 0.683 \\
\hline RVEDA/LVEDA & $42.70(1.30-1405.54)$ & 0.035 \\
\hline OR: odds ratio & & \\
\hline Cl: confidence interval & & \\
\hline BMI: body mass index & \\
\hline LVEF: left ventricular ejection fraction & \\
\hline PEEP: positive end-expiratory pressure & \\
\hline RVFAC: right ventricular fraction area change & \\
\hline RVSP: right ventricular systolic pressure & \\
\hline RVEDA: right ventricular end diastolic area & \\
\hline LVEDA: left ventricular end diastolic area & \\
\hline
\end{tabular}


Table 4

RV dysfunction definition in unadjusted binary logistic regression for longterm development of RV dysfunction

\begin{tabular}{|llll|}
\hline RV dysfunction definition & P Value & OR & $95 \%$ Cl \\
\hline RVD per ASE guidelines & 0.022 & 3.22 & $1.18-8.76$ \\
\hline RVEDA/LVEDA $>0.6+$ Septal dyskinesia & 0.009 & 4.18 & $1.43-12.21$ \\
\hline RVEDA/LVEDA $\geq 1$ & 0.038 & 5.58 & $1.10-28.20$ \\
\hline OR: odds ratio & & & \\
\hline Cl: confidence interval & & & \\
\hline RVD: right ventricle dysfunction & & & \\
\hline RVEDA: right ventricular end diastolic area & & \\
\hline LVEDA: left ventricular end diastolic area & & & \\
\hline
\end{tabular}

\section{Discussion}

Long-term RV dysfunction was noted in nearly half of ARDS survivors who developed RV dysfunction during the course of their ARDS. Logistic regression analysis revealed that a higher RVEDA/LVEDA ratio during ARDS was the only significant risk factor for the occurrence of long-term RV dysfunction in ARDS survivors, while other echocardiographic parameters such as LVEF or TAPSE, or RV fraction area change (RVFAC)/RV systolic pressure (RVSP) (RV-PA coupling) were not found to be statistically significant. In a univariate analysis, RV dysfunction based on the ASE definition, RVEDA/LVEDA ratio > $0.6+$ septal dyskinesia and RVEDA/LVEDA ratio > 1 were also associated with a higher odds of developing long-term $\mathrm{RV}$ dysfunction. This article represents the first study investigating long-term RV function in patients with non-COVID-19 ARDS.

While the data for long-term RV dysfunction in ARDS survivors is sparse. In our study, we found that $50.7 \%$ of patients had long-term RV dysfunction. A meta-analysis of 26 studies (3671 patients) showed that long-term RV dysfunction at median follow-up of 18 months was $18.1 \%$ in survivors of pulmonary embolism [19]. In patients with sub-massive pulmonary embolism, the prevalence of RV dysfunction at 18 months was higher at $40 \%$ illustrating the significant burden of long-term RV dysfunction in critical illness survivors [20]. In a study of survivors of severe ARDS due to coronavirus disease 2019 (COVID-19), $58-78 \%$ were found to have cardiac involvement on cardiac MRI performed a median (IQR) 71 (64-92) days from COVID-19 diagnosis [21]. Huang et al. also showed COVID-19 survivors post-discharge had a significantly reduced RV function compared to controls [22].

Interestingly, Ou et al. found that sepsis survivors had a significantly higher risk of cardiovascular events 1 year after discharge [23]. Similarly, Beesley et al. reported significantly higher cardiovascular events in 
patients with high or low left ventricular global longitudinal systolic strain in sepsis survivors [24]. Unfortunately these studies did not quantify the impact of RV dysfunction in these patients.

The development of RV dysfunction in ARDS is multifaceted. The increase in RV afterload occurs from an imbalance between vasoconstrictors and vasodilators, endothelial injury, hypoxic and hypercapnic vasoconstriction. The increase in pulmonary vascular resistance is further augmented by positive pressure ventilation and positive fluid balance [8]. In addition, primary contractile impairment of RV has also been postulated. Dessap et al. reported that driving pressure $\geq 18 \mathrm{cmH} 2 \mathrm{O}, \mathrm{PaCO} 2 \geq 48 \mathrm{~cm} 2 \mathrm{O}$, and $\mathrm{P} / \mathrm{F}$ ratio $<150 \mathrm{mmHg}$ are independent risk factors of developing RV dysfunction during ARDS [25]. In our study, there was no significant difference in median driving pressure, $\mathrm{PaCO}_{2}$, or $\mathrm{P} / \mathrm{F}$ ratio on the day of echocardiogram between patients who had long-term RV dysfunction and those did not. [26]. This may also explain the absence of any significant difference between the baseline and disease-specific clinical parameters between patients with and without long-term RV dysfunction. Similar to our study, cardiac involvement after recovery from COVID-19 was reported to be independent of preexisting conditions, severity, and overall clinical course [21].

There is a possible physiologic rationale for why we observed an association of RVEDA/LVEDA with longterm RV dysfunction while not detecting similar associations among other markers such as LVEF, TAPSE, $S^{\prime}$, or FAC, which are indicators of myocardial contractility. The inflammatory cytokines and catecholamine associated with critical illness often dysregulate myocardial function, resulting in decreased contractility[27]. These changes are often reversible, normalizing upon recovery from disease $[27,28]$. RVEDA/LVEDA, on the other hand, indicates a severity of RV dysfunction out of proportion to LV dysfunction, including right ventricular failure. While isolated RV enlargement can occur from stress or critical illness, it is much more indicative of high RV afterload than TAPSE, S', or FAC. We observed that the patients with long-term RVD trended towards having higher RV systolic pressure during ARDS (48 vs $28.5 \mathrm{mmHg}, \mathrm{p}=0.07$ ). We speculate that the RVEDA/LVEDA may be more indicative of severe cardiopulmonary disease that might be less likely to recover as rapidly as the impaired contractility seen in critical illness.

"RV-Protective" management strategy in addition to the currently recommended "Lung protective" strategy is currently being suggested to improve outcome in ARDS [29]. It becomes even more important to study the impact of RV protective strategies as RV dysfunction during ARDS not only increases shortterm mortality but may also associated with long-term RV dysfunction as shown in our study [29-31]. Future studies in ARDS and COVID-19 ARDS should not limit the impact of RV protective interventions to short-term outcomes but also consider evaluating the continued benefit of these interventions.

Our study has several important limitations. First, we only included patients who survived ARDS and had a repeat TTE after the discharge. There were likely to be reasons why TTE was performed and this suggests the possibility that included patients might already have a higher risk or prevalence of RV dysfunction than patients without any symptoms or signs. Due to the nature of the retrospective study, it was difficult to completely eliminate this potential selection bias. Second, the complex anatomy of RV 
makes an adequate image-acquirement challenging especially in patients with ARDS. Transesophageal echocardiogram (TEE) was reported to have higher sensitivity to identify RV dysfunction [32], however, the access to TEE is limited in most ICUs. In this study, we have utilized a previously described multimodal definition using TAPSE, S', FAC of RV, semi-qualitative RV size and function as recommended by both the American society of Echocardiography the Preferred Reporting Items for Critical care Echocardiography Studies (PRICES) project endorsed by the European Society of Intensive Care Medicine $[33,34]$ and we believe that we minimized the risk of missing RV dysfunction by using these standardized echocardiographic parameters. Third, this was a single-center small study and the generalizability of our findings needs to be clarified with larger studies. Last, our regression model's limited sample size may be underpowered to detect some associations. Given the small sample size at a single-center and potential selection bias due to retrospective nature, a larger prospective study investigating long-term RV function in ARDS survivors to enable our knowledge of causation and association to long-term clinical outcome including mortality is warranted.

\section{Conclusions}

Right ventricular dysfunction is very common among patients with ARDS, and as we demonstrated it can persist long-term in half of the survivors. An increased RVEDA/LVEDA during ARDS was found to be a significant risk factor for long-term RV dysfunction, while other echocardiographic parameters such as LVEF or TAPSE were not. Our study highlights the need for a cardiovascular examination including an echocardiogram in ARDS survivors to understand the true prevalence of long-term RV dysfunction and its impact on patient-centered outcomes.

\section{Abbreviations}

$\mathrm{RV}$ : right ventricle / right ventricular

RVD: right ventricular dysfunction

ARDS: acute respiratory distress syndrome

ICU: intensive care unit

TTE: transthoracic echocardiography

TEE: transesophageal echocardiography

OR: odds ratio

Cls: confidence intervals

PEEP: positive end-expiratory pressure 
RVEDA: right ventricular end-diastolic area

LVEDA: left ventricular end-diastolic area

TAPSE: tricuspid annular plane systolic excursion

TV TDI S': peak systolic velocity at the tricuspid valve annulus

ACP: acute cor pulmonale

VV-ECMO: veno-venous extracorporeal membrane oxygenation

\section{Declarations}

Author Contributions: S.D., R.S., H.J. and A.D. conceived and designed the study. S.D., R.S., and H.J., acquired the data. S.D., R.S., H.J., X.W., X.H., and A.D. conducted the analysis. S.D. S.D., R.S., H.J.X.W., X.H., V.Z., M.L. and A.D drafted the manuscript. All authors revised it critically for important intellectual content and gave final approval of the version to be published: all authors. Agreement to be accountable for all aspects of the work in ensuring that questions related to the accuracy or integrity of any part of the work are appropriately investigated and resolved. S.D. and A.D. accept responsibility to being guarantor of the paper, taking responsibility for the integrity of the work as a whole, from inception to published article.

\section{Financial/nonfinancial disclosures}

The present work was performed without any direct or indirect financial support.

\section{Conflict of interest statements:}

S.D., R.S., H.J., X.H., X.W., V.Z., M.J. and A.D. have no conflict of interest.

\section{IRB Approval}

This study was conducted in accordance with the amended Declaration of Helsinki. Local institutional review boards (IRB\#19-1200) approved the protocol, and informed consent was obtained from all patients or their appropriate healthcare representative. A patient information sheet was provided to each patient or their healthcare representative as per local IRB recommendation.

\section{Acknowledgements}

None

\section{Consent for publication}

All authors consent to publication of manuscript and support material. 
This study was approved by the Institutional Review Committee of Cleveland Clinic (IRB\#19-1200).

\section{Availability of data and materials}

The datasets used and/or analyzed during the current study are available from the corresponding author on reasonable request.

\section{References}

1. Bellani G, Laffey JG, Pham T, Fan E, Brochard L, Esteban A, Gattinoni L, Haren Fv, Larsson A, McAuley DF, et al. Epidemiology, Patterns of Care, and Mortality for Patients With Acute Respiratory Distress Syndrome in Intensive Care Units in 50 Countries. JAMA. 2016;315(8):788-800.

2. Zhang Z, Spieth PM, Chiumello D, Goyal H, Torres, Antoni, Laffey JG, Hong Y. Declining Mortality in Patients With Acute Respiratory Distress Syndrome: An Analysis of the Acute Respiratory Distress Syndrome Network Trials. Critical care medicine 2019, 47(3).

3. Zambon M, Vincent J-L. Mortality Rates for Patients With Acute Lung Injury/ARDS Have Decreased Over Time. Chest. 2008;133(5):1120-7.

4. Jardin F, Fellahi JL, Beauchet A, Vieillard-Baron A, Loubières $Y$, Page B. Improved prognosis of acute respiratory distress syndrome 15 years on. Intensive Care Med. 1999;25(9):936-41.

5. Maca J, Jor O, Holub M, Sklienka P, Burda F, Janout M. V, P: Past and Present ARDS Mortality Rates: A Systematic Review. Respir Care. 2017;62(1):113-22.

6. Herridge MS, Cheung AM, Tansey CM, Matte-Martyn A, Diaz-Granados N, Al-Saidi F, Cooper AB, Guest $\mathrm{CB}$, Mazer CD, Mehta S, et al. One-year outcomes in survivors of the acute respiratory distress syndrome. N Engl J Med. 2003;348(8):683-93.

7. Herridge MS, Tansey CM, Matté A, Tomlinson G, Diaz-Granados N, Cooper A, Guest CB, Mazer CD, Mehta S, Stewart TE, et al. Functional disability 5 years after acute respiratory distress syndrome. N Engl J Med. 2011;364(14):1293-304.

8. Zochios V, Parhar K, Tunnicliffe W, Roscoe A, Gao F. The Right Ventricle in ARDS. Chest. 2017;152(1):181-93.

9. Vieillard-Baron A. Is right ventricular function the one that matters in ARDS patients? Definitely yes. In: Applied Physiology in Intensive Care Medicine 2. 2012: 397-399.

10. Mekontso Dessap A, Boissier F, Charron C, Begot E, Repesse X, Legras A, Brun-Buisson C, Vignon P, Vieillard-Baron $A$. Acute cor pulmonale during protective ventilation for acute respiratory distress syndrome: prevalence, predictors, and clinical impact. Intensive Care Med. 2016;42(5):862-70.

11. Sato R, Dugar S, Cheungpasitporn W, Schleicher M, Collier P, Vallabhajosyula S, Duggal A. The impact of right ventricular injury on the mortality in patients with acute respiratory distress syndrome: a systematic review and meta-analysis. Crit Care (London England). 2021;25(1):172.

12. Kjaergaard J, Akkan D, Iversen KK, Kober L, Torp-Pedersen C, Hassager C. Right ventricular dysfunction as an independent predictor of short- and long-term mortality in patients with heart 
failure. Eur J Heart Fail. 2007;9(6-7):610-6.

13. Panitchote A, Mehkri O, Hastings A, Hanane T, Demirjian S, Torbic H, Mireles-Cabodevila E, Krishnan $S$, Duggal A. Factors associated with acute kidney injury in acute respiratory distress syndrome. Ann Intensive Care. 2019;9(1):74.

14. Vallabhajosyula S, Shankar A, Vojjini R, Cheungpasitporn W, Sundaragiri PR, Dubrock HM, Sekiguchi H, Frantz RP, Cajigas HR, Kane GC, et al. Impact of Right Ventricular Dysfunction on Short-term and Long-term Mortality in Sepsis. Chest. 2021;159(6):2254-63.

15. Lang RM, Badano LP, Mor-Avi V, Afilalo J, Armstrong A, Ernande L, Flachskampf FA, Foster E, Goldstein SA, Kuznetsova T, et al. Recommendations for cardiac chamber quantification by echocardiography in adults: an update from the American Society of Echocardiography and the European Association of Cardiovascular Imaging. Eur Heart J Cardiovasc Imaging. 2015;16(3):23370.

16. Lang RM, Badano LP, Mor-Avi V, Afilalo J, Armstrong A, Ernande L, Flachskampf FA, Foster E, Goldstein SA, Kuznetsova T, et al. Recommendations for Cardiac Chamber Quantification by Echocardiography in Adults: An Update from the American Society of Echocardiography and the European Association of Cardiovascular Imaging. J Am Soc Echocardiogr. 2015;28(1):1-39.e14.

17. Vallabhajosyula S, Kumar M, Pandompatam G, Sakhuja A, Kashyap R, Kashani K, Gajic O, Geske JB, Jentzer JC. Prognostic impact of isolated right ventricular dysfunction in sepsis and septic shock: an 8-year historical cohort study. Ann Intensive Care. 2017;7(1):94.

18. Stekhoven DJ, Buhlmann P. MissForest-non-parametric missing value imputation for mixed-type data. Bioinformatics. 2012;28(1):112-8.

19. Sista AK, Miller LE, Kahn SR, Kline JA. Persistent right ventricular dysfunction, functional capacity limitation, exercise intolerance, and quality of life impairment following pulmonary embolism: Systematic review with meta-analysis. Vasc Med. 2017;22(1):37-43.

20. Konstantinides SV, Vicaut E, Danays T, Becattini C, Bertoletti L, Beyer-Westendorf J, Bouvaist H, Couturaud F, Dellas C, Duerschmied D, et al. Impact of Thrombolytic Therapy on the Long-Term Outcome of Intermediate-Risk Pulmonary Embolism. J Am Coll Cardiol. 2017;69(12):1536-44.

21. Puntmann VO, Carerj ML, Wieters I, Fahim M, Arendt C, Hoffmann J, Shchendrygina A, Escher F, VasaNicotera M, Zeiher AM, et al: Outcomes of Cardiovascular Magnetic Resonance Imaging in Patients Recently Recovered From Coronavirus Disease 2019 (COVID-19). JAMA Cardiol 2020, 5(11):12651273.

22. Huang L, Zhao P, Tang D, Zhu T, Han R, Zhan C, Liu W, Zeng H, Tao Q, Xia L. Cardiac Involvement in Patients Recovered From COVID-2019 Identified Using Magnetic Resonance Imaging. JACC Cardiovasc Imaging. 2020;13(11):2330-9.

23. Ou SM, Chu H, Chao PW, Lee YJ, Kuo SC, Chen TJ, Tseng CM, Shih CJ, Chen YT. Long-Term Mortality and Major Adverse Cardiovascular Events in Sepsis Survivors. A Nationwide Population-based Study. Am J Respir Crit Care Med. 2016;194(2):209-17. 
24. Beesley SJ, Sorensen J, Walkey AJ, Tonna JE, Lanspa MJ, Hirshberg E, Grissom CK, Horne BD, Burk R, Abraham TP, et al. Long-Term Implications of Abnormal Left Ventricular Strain During Sepsis. Critical care medicine. 2021;49(4):e444-53.

25. Mekontso Dessap A, Boissier F, Charron C, Bégot E, Repessé X, Legras A, Brun-Buisson C, Vignon P, Vieillard-Baron A. Acute cor pulmonale during protective ventilation for acute respiratory distress syndrome: prevalence, predictors, and clinical impact. Intensive care medicine. 2016;42(5):862-70.

26. Wang CY, Calfee CS, Paul DW, Janz DR, May AK, Zhuo H, Bernard GR, Matthay MA, Ware LB, Kangelaris KN. One-year mortality and predictors of death among hospital survivors of acute respiratory distress syndrome. Intensive Care Med. 2014;40(3):388-96.

27. Beesley SJ, Weber G, Sarge T, Nikravan S, Grissom CK, Lanspa MJ, Shahul S, Brown SM. Septic Cardiomyopathy. Crit Care Med. 2018;46(4):625-34.

28. Etchecopar-Chevreuil C, François B, Clavel M, Pichon N, Gastinne H, Vignon P. Cardiac morphological and functional changes during early septic shock: a transesophageal echocardiographic study. Intensive Care Med. 2008;34(2):250-6.

29. Paternot A, Repesse X, Vieillard-Baron A. Rationale and Description of Right Ventricle-Protective Ventilation in ARDS. Respir Care. 2016;61(10):1391-6.

30. Vieillard-Baron A, Charron C, Caille V, Belliard G, Page B, Jardin F. Prone positioning unloads the right ventricle in severe ARDS. Chest. 2007;132(5):1440-6.

31. Zochios V, Parhar K, Vieillard-Baron A. Protecting the Right Ventricle in ARDS: The Role of Prone Ventilation. J Cardiothorac Vasc Anesth. 2018;32(5):2248-51.

32. Lheritier G, Legras A, Caille A, Lherm T, Mathonnet A, Frat JP, Courte A, Martin-Lefevre L, Gouello JP, Amiel JB, et al. Prevalence and prognostic value of acute cor pulmonale and patent foramen ovale in ventilated patients with early acute respiratory distress syndrome: a multicenter study. Intensive Care Med. 2013;39(10):1734-42.

33. Huang S, Sanfilippo F, Herpain A, Balik M, Chew M, Clau-Terre F, Corredor C, De Backer D, Fletcher N, Geri G, et al. Systematic review and literature appraisal on methodology of conducting and reporting critical-care echocardiography studies: a report from the European Society of Intensive Care Medicine PRICES expert panel. Ann Intensive Care. 2020;10(1):49.

34. Sanfilippo F, Huang S, Herpain A, Balik M, Chew MS, Clau-Terré F, Corredor C, De Backer D, Fletcher N, Geri G, et al. The PRICES statement: an ESICM expert consensus on methodology for conducting and reporting critical care echocardiography research studies. Intensive care medicine. 2021;47(1):1-13.

\section{Figures}




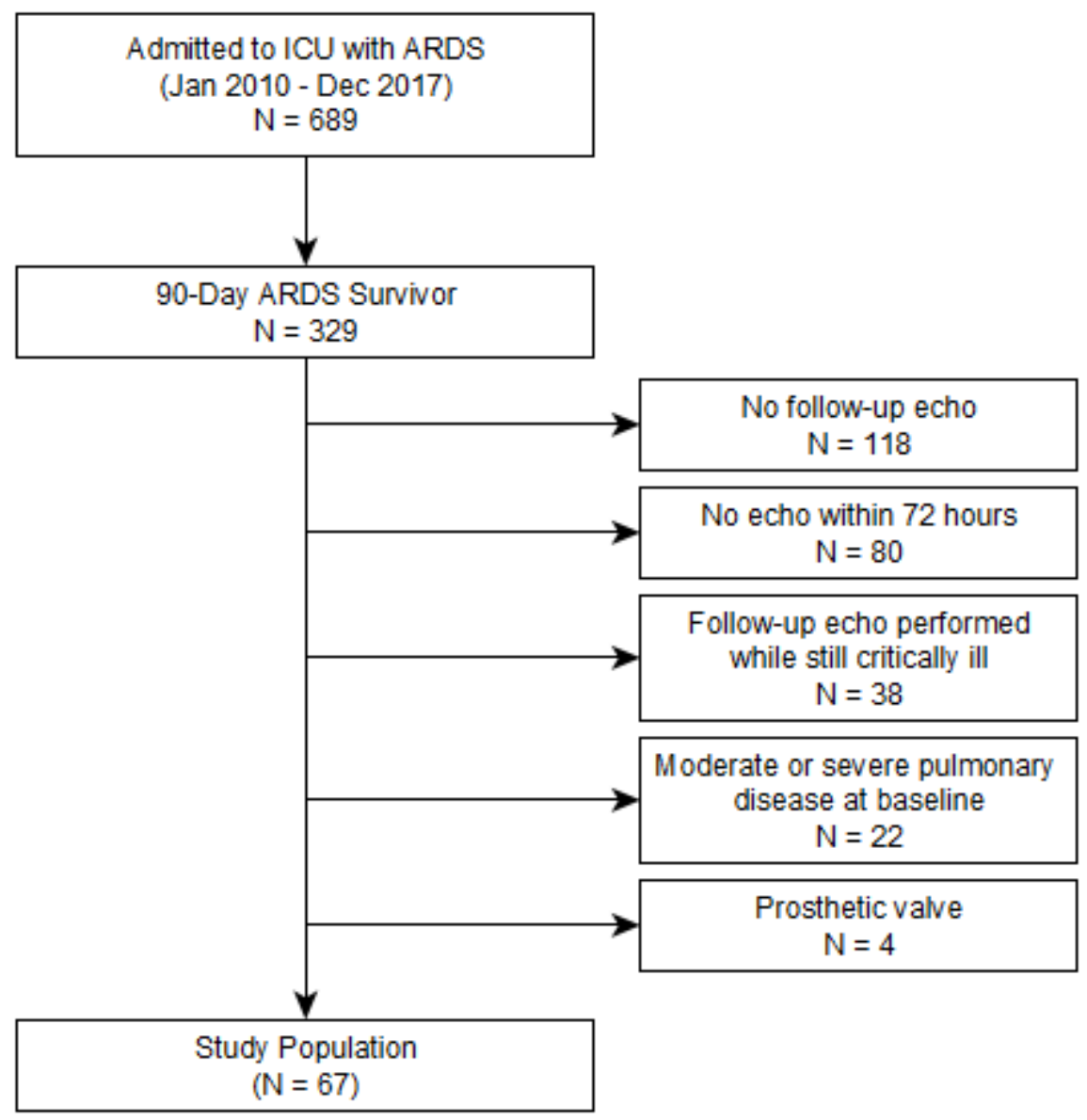

Figure 1

Consort flow chart

\section{Supplementary Files}

This is a list of supplementary files associated with this preprint. Click to download.

- GraphicalAbstract.jpg 